\title{
A multi-task graph-clustering approach for chromosome conformation capture data sets identifies conserved modules of chromosomal interactions
}

\author{
Alireza Fotuhi Siahpirani ${ }^{1}$, Ferhat $\mathrm{Ay}^{2}$ and Sushmita Roy ${ }^{3,4^{*}}$
}

\begin{abstract}
Chromosome conformation capture methods are being increasingly used to study three-dimensional genome architecture in multiple cell types and species. An important challenge is to examine changes in three-dimensional architecture across cell types and species. We present Arboretum-Hi-C, a multi-task spectral clustering method, to identify common and context-specific aspects of genome architecture. Compared to standard clustering, Arboretum-Hi-C produced more biologically consistent patterns of conservation. Most clusters are conserved and enriched for either high- or low-activity genomic signals. Most genomic regions diverge between clusters with similar chromatin state except for a few that are associated with lamina-associated domains and open chromatin.
\end{abstract}

\section{Background}

The three-dimensional (3D) organization of the genome is emerging as an important layer in the regulation of gene expression [1-10]. Recent advances in high-throughput chromosome conformation capture (3C, particularly $4 \mathrm{C}$, $5 \mathrm{C}$, and $\mathrm{Hi}-\mathrm{C}$ ) technology allow us to examine the $3 \mathrm{D}$ organization of a genome in an unbiased and comprehensive manner $[1,8]$. Genome-wide $3 \mathrm{C}$ data sets are becoming increasingly available for multiple species and tissues and have enabled us to examine the folding and organizational principles of the genome and identify longrange interactions among genomic loci $[1,11]$. In particular, studies in yeast have shown that such long-range interactions are enriched for loci involving tRNA genes, centromeres, early origins of replication [4], and transcription factories for regulation of gene expression [12]. In mammalian systems, such interactions are organized into architectural units known as compartments and

*Correspondence: sroy@biostat.wisc.edu

3 Wisconsin Institute for Discovery, University of Wisconsin, Madison, WI 53717 , USA

${ }^{4}$ Department of Biostatistics and Medical Informatics, University of Wisconsin, Madison, WI 53717, USA

Full list of author information is available at the end of the article topologically associated domains (TADs). While the interactions can be cell-type- [13] or species-specific [14, 15], the compartments and TADs are likely conserved across developmental stages $[3,16]$ and across species [14]. However, our understanding of the extent of conservation and context-specificity of these interactions is incomplete.

The availability of genome-wide $3 \mathrm{C}$ data sets for multiple species and tissues gives us the unique opportunity to compare chromatin organization across tissues and organisms to identify the principles of this organization. In parallel, statistical techniques have been developed to normalize these data, identify significant interacting genomic loci [17-19], and identify different types of organizational units from these data [20]. Clustering and dimensionality reduction approaches, in particular, have emerged as important analytical tools for $\mathrm{Hi}-\mathrm{C}$ data $[8,9,19,21]$. Rao et al. clustered high-resolution in situ $\mathrm{Hi}-\mathrm{C}$ data and found six main clusters exhibiting distinct patterns of chromatin state [9]. Principal component analyses of $\mathrm{Hi}-\mathrm{C}$ data for each chromosome revealed a compartment structure [8], where regions within each compartment are more likely to interact than regions from two different compartments. Imakaev et al. found that the first eigenvector of the genome-wide normalized contact count map exhibited similar properties as 
the two-compartment model [21]. The second and third eigenvectors exhibited variation along the chromosomal arms, with increased magnitude in the centromeric and telomeric regions for the second and third eigenvectors, respectively.

While current clustering and dimensionality reduction techniques have provided useful insights into genome organization, there are several key issues that need to be addressed. First, unlike traditional functional genomics data such as genome-wide mRNA level or histone modification measurements, 3C data specify contact counts among pairs of genomic loci. A graph-based representation provides a natural representation of these $\mathrm{Hi}-\mathrm{C}$ data [22] and incorporating $\mathrm{Hi}-\mathrm{C}$ interaction information as a graph prior was recently shown to improve chromatinmark-based genome segmentation and annotation [23]. Graph-clustering methods, such as spectral clustering $[24,25]$, when applied to graph data, are more advantageous than using conventional clustering. However, to our knowledge, graph-clustering methods, especially across multiple cell types and species, have not been explored with $\mathrm{Hi}-\mathrm{C}$ data. It is currently unknown whether such methods have any advantages over traditional clustering methods that do not capture the graph nature of $3 \mathrm{C}$ data.

The second issue is that methods that systematically compare these maps across multiple tissues or multiple organisms are scarce [3]. In particular, given such contact count matrices from two or more cell types, tissues, or organisms, it is not immediately clear how to identify clusters simultaneously in both cell types and also compare them to identify common and context-specific patterns. The systematic comparison of the general 3D organization of the genome across multiple conditions, cell types, and organisms is still a largely unexplored computational challenge.

In this paper, we first perform a comprehensive analysis of different clustering approaches (hierarchical, $k$-means, and spectral) using different distance measures. Our analysis shows that spectral clustering methods tend to outperform existing non-graph-based methods, producing higher quality clusters based on statistical enrichment of multiple one-dimensional regulatory genomic signals. We next develop a multi-task version of our spectral clustering algorithm and apply it to $\mathrm{Hi}-\mathrm{C}$ data in four cell lines, two each from human and mouse. Compared to an independent clustering method, our multi-task clustering method finds more biologically consistent patterns of conservation and divergence. Using the inferred clusters, we perform a systematic comparative study of the extent of conservation and divergence in chromosome contact preferences between matched cell lines of different species, and between cell lines of the same species. Our results indicate that most regions maintain their chromosome contact preferences between cell lines, and regions that diverge between species and cell lines are enriched for lamina-associated domains (LADs) and architectural proteins.

\section{Results}

\section{Graph-based clustering of $\mathrm{Hi}-\mathrm{C}$ data recovers better} clusters than non-graph-based clustering

To assess the utility of graph-based clustering for $\mathrm{Hi}-\mathrm{C}$ data over non-graph-based clustering, we compared three algorithms: (1) hierarchical clustering, (2) $k$-means, and (3) spectral clustering. Hierarchical clustering and $k$-means have been used widely to analyze functional genomics data sets such as gene expression [26] and chromatin marks [27]. The spectral clustering algorithm is a graph-based clustering method that clusters the eigenvectors of the Laplacian operator on a graph [25]. For all three clustering methods, we considered different distance metrics: (1) Euclidean distance, (2) Pearson's correlation, (3) Spearman's correlation, (4) contact counts, and (5) $\log _{2}$ of contact counts. In total, we had 15 clustering approaches that differed by clustering algorithm and distance metric.

We applied each clustering method to $\mathrm{Hi}-\mathrm{C}$ data from the human $\mathrm{H} 1$ embryonic stem cell (hESC) line [3], binned into 2755 1-Mbp bins. Each method was applied to obtain $k=10$ clusters (Methods). We evaluated the quality of clusters from each clustering method using five different statistical measures: (1) the Davies-Bouldin index (DBI), (2) the silhouette index (SI), (3) the difference in contact counts between regions in the same cluster and between regions from different clusters (delta contact count), (4) the number of clusters enriched for a regulatory signal (e.g. transcription factor occupancy or histone modification), and (5) analysis of variance (ANOVA) of a regulatory signal. DBI measures the within-cluster scatter and is a number between 0 and 1 ; the lower the value the better the clustering. SI assesses the boundaries of clustering and ranges between -1 and 1 ; the lower the value the worse the clustering. The Kolmogorov-Smirnov (KS) test was used to assess whether a particular feature was significantly high in a cluster compared to the genomic background. ANOVA was used to examine how well the clusters explain the variation in a particular regulatory signal. DBI, SI, and the delta contact count served as internal validation metrics of clustering that need only the data being clustered, while the number of enriched clusters and ANOVA served as measures of external validation.

A comparison of different clustering approaches showed considerable variation among the different methods (Fig. 1). For example, using DBI and SI, hierarchical clustering with 1-Pearson's correlation as a distance measure was among the best performing methods (Fig. 1a, b), but it was among the worst when using the number 

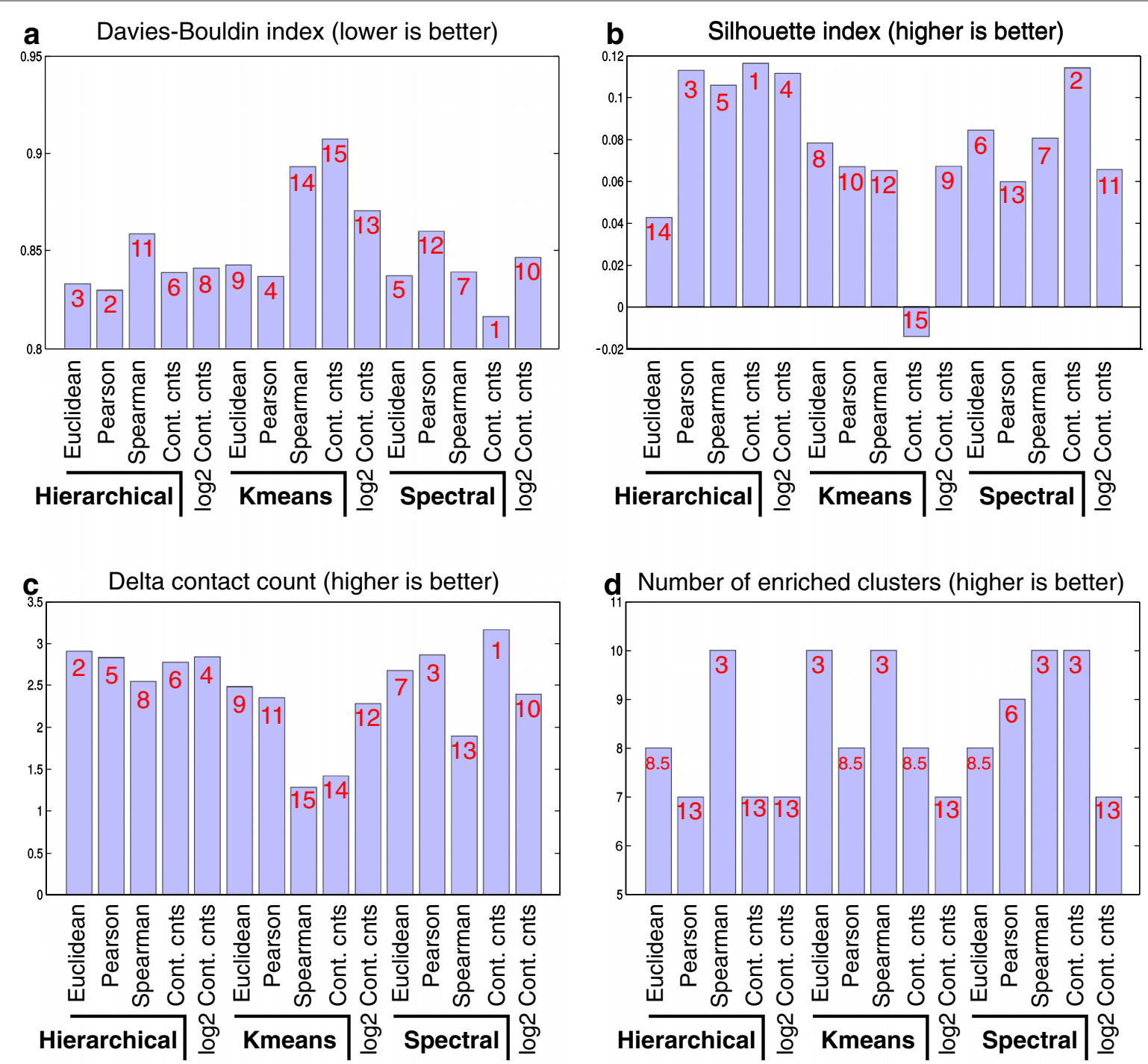

d Number of enriched clusters (higher is better)

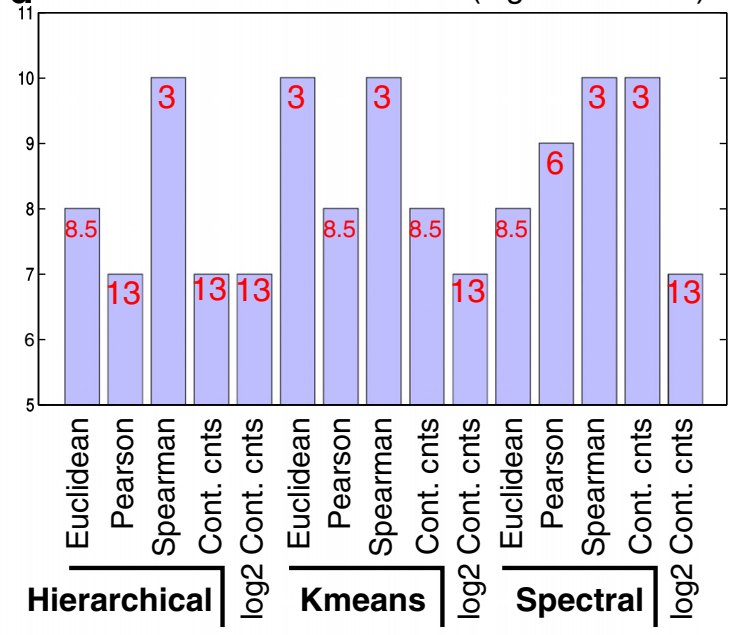

e - $\log$ ANOVA p-values

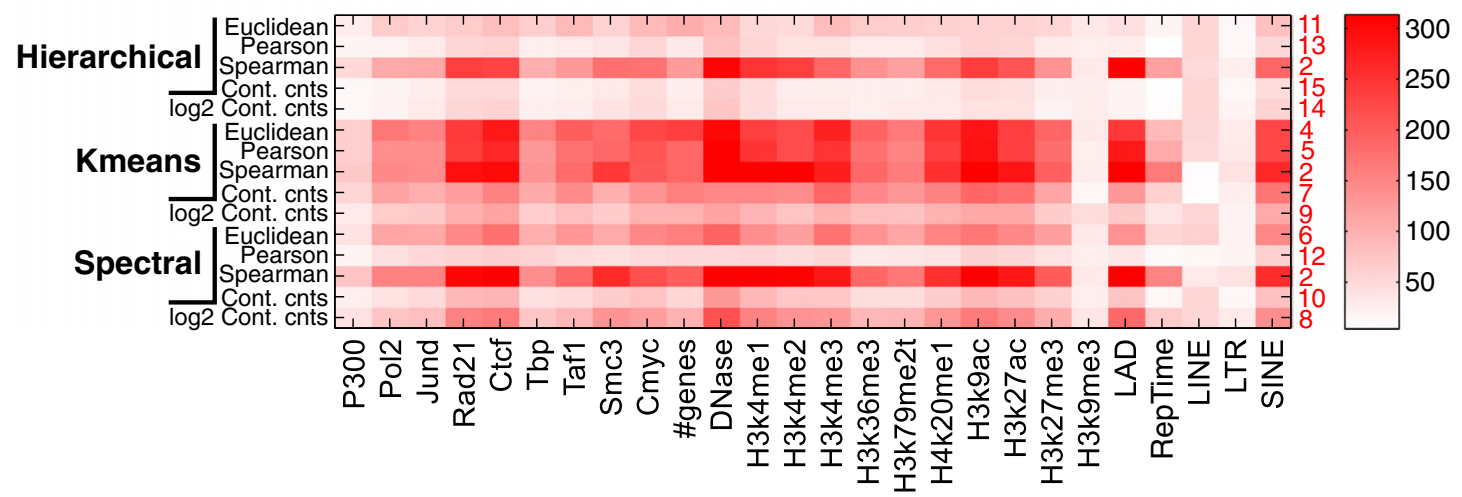

Fig. 1 Evaluation of different clustering methods. Shown are a comparison of three clustering methods using different measures: a Davies-Bouldin index. b Silhouette index. c Difference in contact counts within and between clusters. $\mathbf{d}$ Number of clusters with an enriched regulatory signal. e $-\log P$ value of ANOVA for different regulatory signals. Each clustering method was applied with five different distance/similarity criteria between genomic regions: Euclidean, Pearson's correlation, Spearman's correlation, contact count, and $\log _{2}$ of contact count. The numbers (in red) correspond to the rank of the methods using the given measure. Cont. cnts contact count 
of enriched clusters or ANOVA (Fig. 1d, e). To compare the different clustering approaches across all these measures, we, therefore, ranked each method on a scale of 1 to 15 (appropriately adjusting ties) on each of the evaluation metrics, and computed the average rank for each method. Based on the average rank, the top five methods were spectral clustering on contact count (1), hierarchical clustering with 1-Spearman's correlation as the distance measure (2), spectral clustering with Spearman's correlation (3), spectral clustering with Euclidean distance (4), and $k$ means using Euclidean distance (5). Thus, three among the top five ranking methods were spectral clustering variants. We next inspected the patterns of enrichment in the clusters from each method. We found that clusters obtained from spectral clustering with Spearman's correlation (Fig. 2) were most distinct in their patterns of enrichment compared to the other variants of spectral clustering (Additional file 1: Figure S1) and hierarchical clustering (Additional file 1: Figure S2, Additional file 2). In particular, spectral clustering with Spearman's correlation found three clusters that were significantly enriched with open chromatin signatures (described in detail in the next section, Fig. 2d). In contrast, clusters from hierarchical clustering were unbalanced and all the activating marks were concentrated in one cluster. Thus, the clusters obtained from spectral clustering on Spearman's correlation are likely more biologically meaningful based on external validation measures and are comparable to hierarchical clustering approaches for internal validation metrics. Based on these observations, we selected spectral clustering (Spearman's correlation) for our subsequent analysis. We note that our clustering framework is flexible and can use other definitions of graph weights as well.

\section{Spectral clustering can incorporate both cis and trans interactions and identifies two major types of clusters}

Inspection of the chromosomal coverage of our clusters in hESC showed that most (six of ten) clusters cover multiple chromosomes, revealing cis and trans interactions (Fig. 2a, Methods). Spectral clustering of only trans interactions finds a similar number of multi-chromosomal clusters suggesting that our clustering is robust and that intrachromosomal interactions do not overshadow the interchromosomal interactions (Additional file 1: Figure S3, Additional file 2).

To interpret our clusters functionally and relate them to downstream gene expression programs, we tested our clusters for statistical enrichment of multiple genome-wide regulatory signals including chromatin marks (H3K4me1, H3K4me2, H3K4me3, H3K36me3, H3K79me2, H4K20me1, H3K9ac, H3K27ac, H3K27me3, and $\mathrm{H} 3 \mathrm{~K} 9 \mathrm{me} 3)$, LADs, early versus late replication timing (RepTime), general transcription factors (POLII, TAF, TBP, CTCF, P300, and CMYC), cohesin components
(RAD21 and SMC3), open chromatin from DNase I hypersensitivity assays, number of genes, and various classes of repeat elements [short interspersed nuclear elements (SINEs), long interspersed elements (LINEs), and long terminal repeats (LTRs)].

We found that clusters $\mathrm{C} 0, \mathrm{C} 1$, and $\mathrm{C} 2$ were significantly enriched with gene-rich regions, open chromatin (DNase I), SINE, and activating and repressive marks, with the exception of H3K9me3, which varied between the clusters (KS test $P<0.05$, Fig. 2d, e). Cluster C0 was also moderately enriched for LADs while $\mathrm{C} 1$ and $\mathrm{C} 2$ were depleted in LADs. The remaining seven clusters were associated with LADs, LINEs, and LTRs, and were depleted for genes and chromatin marks. The clusters comprising entirely regions from one chromosome were associated with LADs and either LINE (C7 and C8) or LTRs (C6). We also observed that SINE and LINE enrichments are exclusive: SINEs tend to be with clusters with high genomic activity (i.e. enriched for different chromatin marks and gene-rich regions), while LINE and LTR elements are associated with LAD clusters. Our observation that the clusters associated with gene-rich regions are depleted in LADs and clusters associated with genepoor regions are enriched for LADs is in agreement with previous studies that showed LADs are relatively gene poor [28]. Because the clusters appeared to be discriminated based on activity, we asked if DNase I footprints can explain the association of all other marks. We observe significant conditional mutual information between each signal and the clustering assignments given DNase I, which suggests there is information to be gained by the clustering that is not captured in the DNase I signal (Additional file 1: Methods, Additional file 1: Figure S4). Furthermore, the observed values of the different evaluation metrics (SI, DBI, and delta contact counts) are significantly higher than random, suggesting that we are not over-clustering (Additional file 1: Methods and Additional file 1: Figure S5).

In parallel, we clustered the genomic regions using $k$ means on their one-dimensional signal profiles (Fig. 2f) and compared these clusters to the spectral clusters based on a hypergeometric test. Several of the Hi-C clusters were mutually enriched in these $k$-means clusters (Fig. $2 \mathrm{~g}$ ), suggesting that these two partitions of the data are mutually consistent with each other. For example, the spectral clusters $\mathrm{C} 1$ and $\mathrm{C} 2$ (with high genomic activity) had significant overlap with the $k$-means clusters $\mathrm{C} 0$ and $\mathrm{C} 4$. However, the Hi-C clusters do not have a one-to-one mapping with the one-dimensional signal $k$-means clusters (e.g. the C3 $k$-means cluster had significant overlap with the $\mathrm{C} 4, \mathrm{C} 6$, and $\mathrm{C} 7$ spectral clusters), suggesting that the $\mathrm{Hi}-\mathrm{C}$ clusters capture additional information that is specific to the 3D organization of the genome. We repeated this analysis for Hi-C data in a mouse ESC (mESC) line 


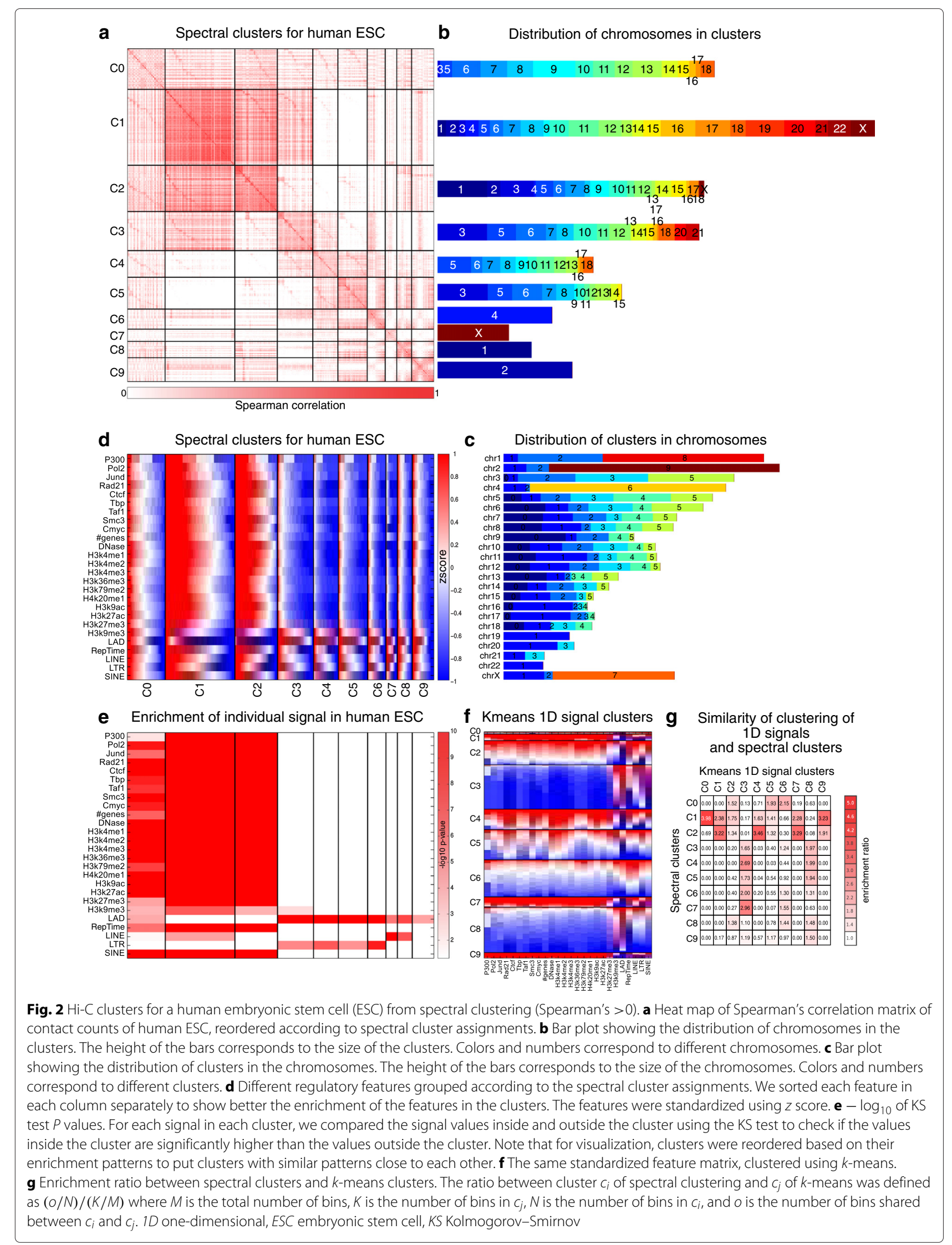


from Dixon et al. [3] (Additional file 1: Figure S6 and Additional file 2), and observed similar patterns, suggesting that our clusters are capturing generalizable properties of chromosomal organization.

To test the sensitivity of our conclusions to fixed-sized bins, we also considered regions defined by TADs. Briefly, we aggregated the counts in TADs defined in Dixon et al. [3] and clustered the resulting matrix (Additional file 1: Methods). We observed similar patterns of enrichment in these clusters and found that $43 \%$ of the total bases were co-clustered when using a fixed bin size and clusters of TADs (Additional file 1: Figure S7, Additional file 2). We also repeated our analysis of the hESC data for multiple resolutions, 100 and $500 \mathrm{kbp}$. There was a significant overlap of base pair coverage between clusters at different resolutions (64 \% for 100 and $500 \mathrm{kbp}, 53 \%$ for $100 \mathrm{kbp}$ and $1 \mathrm{Mbp}$, and $71 \%$ for $500 \mathrm{kbp}$ and $1 \mathrm{Mbp}$ ), which is significantly greater than random (Additional file 1: Table S1). Furthermore, we could find a one-to-one mapping for the majority of the clusters, and the mapped clusters also exhibited similar patterns of enrichment as the 1-Mbp regions (Additional file 1: Figure S8).

\section{$\mathrm{Hi}-\mathrm{C}$ data clusters from spectral clustering recapitulate known and novel higher-order organizational units}

To examine the relationship between our spectral clusters and major chromosomal architectural units such as compartments on individual chromosomes [8], we applied $k=2$ clustering to our data. A compartment is defined by a subset of regions on a chromosome that densely interact with each other, but are depleted for interactions with other regions on the chromosome. We obtained the cluster assignment for all regions in a chromosome and compared these cluster assignments to the compartments (Additional file 1: Figure S9 and Methods). The majority of the chromosomes (except for chromosomes 16, 19, 20,21 , and 22) were partitioned into two clusters by our approach, indicating the presence of compartment-like structures in our clustering results. Pairs of regions that were clustered together by spectral clustering tended to be in the same compartment as assessed by two independent measures of co-clustering. In the majority of the chromosomes (18 out of 23 ), these measures are significantly higher than what is expected by chance ( $F$ score: $60-80 \%$, $t$ test $P<3.49 \times 10^{-5}$, and Rand index: $50-80 \%, t$ test $P<$ $1.45 \times 10^{-5}$, Additional file 1: Figure S9), suggesting that spectral clustering with $k=2$ can also recover aspects of compartments. Chromosomes 16, 19, 20, 21, and 22 are not detectable as separate clusters with $k=2$, likely because they tend to co-localize in the nucleus [8]. The application of the spectral clustering method at higher resolution (e.g. $40 \mathrm{kbp}$ instead of $1 \mathrm{Mbp}$ ), can recover TAD-like structures (Additional file 1: Figure S10a, b, c, $\mathrm{d}$, and Additional file 1: Methods). In addition, applying the clustering method to each chromosome separately can also recover clusters with significant overlap with the compartment (Additional file 1: Figure S10e, f, g). These results further suggest that graph-based clustering approaches can be a general and powerful approach for recovering different organizational units of the genome, spanning both cis (within one chromosome) and trans (between chromosome) interactions.

\section{Arboretum-Hi-C: A multi-task spectral clustering algorithm for comparative analysis of $\mathrm{Hi}-\mathrm{C}$ data}

Having determined that spectral clustering is a powerful approach for analyzing $\mathrm{Hi}-\mathrm{C}$ data from one cell line, we next developed a new approach, Arboretum-Hi-C, to compare systematically the $3 \mathrm{D}$ organization across multiple cell types and species. Arboretum-Hi-C combines two clustering strategies: spectral clustering and multitask clustering (Fig. 3). Multi-task clustering is a special case of multi-task learning [29], where the goal is to solve multiple learning tasks simultaneously. Arboretum-Hi-C takes as input $n$ different Hi-C data sets ( $n=3$ in Fig. 3), representing possibly different cell lines or species, a tree describing the hierarchical relationship between the data sets, the number of clusters $k$, and a mapping of regions between the different data sets. The Hi-C data sets represent observed data as the leaves of the tree (Fig. 3). As output, Arboretum-Hi-C returns the cluster assignments of regions in each $\mathrm{Hi}-\mathrm{C}$ data set. Arboretum-Hi-C is based on a previous multi-task clustering approach, Arboretum [30], which uses a generative probabilistic model to cluster expression data from multiple species while accounting for the hierarchical relationships among the species as described by a phylogenetic tree (Methods). However, instead of expression matrices at each leaf node, we now have $\mathrm{Hi}-\mathrm{C}$ interaction graphs. Edges in these graphs are weighted, with edge weights corresponding to Spearman's correlation since this gave the best results among different distance metrics. However, our general approach is applicable to different definitions of edge weight (e.g. contact count between a pair of regions). To cluster these graphs, we apply Gaussian mixture model-based clustering to the first $k$ eigenvectors of each graph's Laplacian (Additional file 1: Methods).

\section{Major modules of chromosome contact interactions are shared between human and mouse cell lines}

We applied Arboretum-Hi-C to two human and two mouse cell lines that were studied in Dixon et al. [3]. Two of these cell lines represent the undifferentiated ESC state in both organisms (hESC and mESC, respectively), and the other two cell lines represent examples of a terminally differentiated cell state (IMR90 human fibroblasts and mouse cortex, referred to as hIMR90 and mCortex, respectively). We first examined 1318 1-Mbp human 


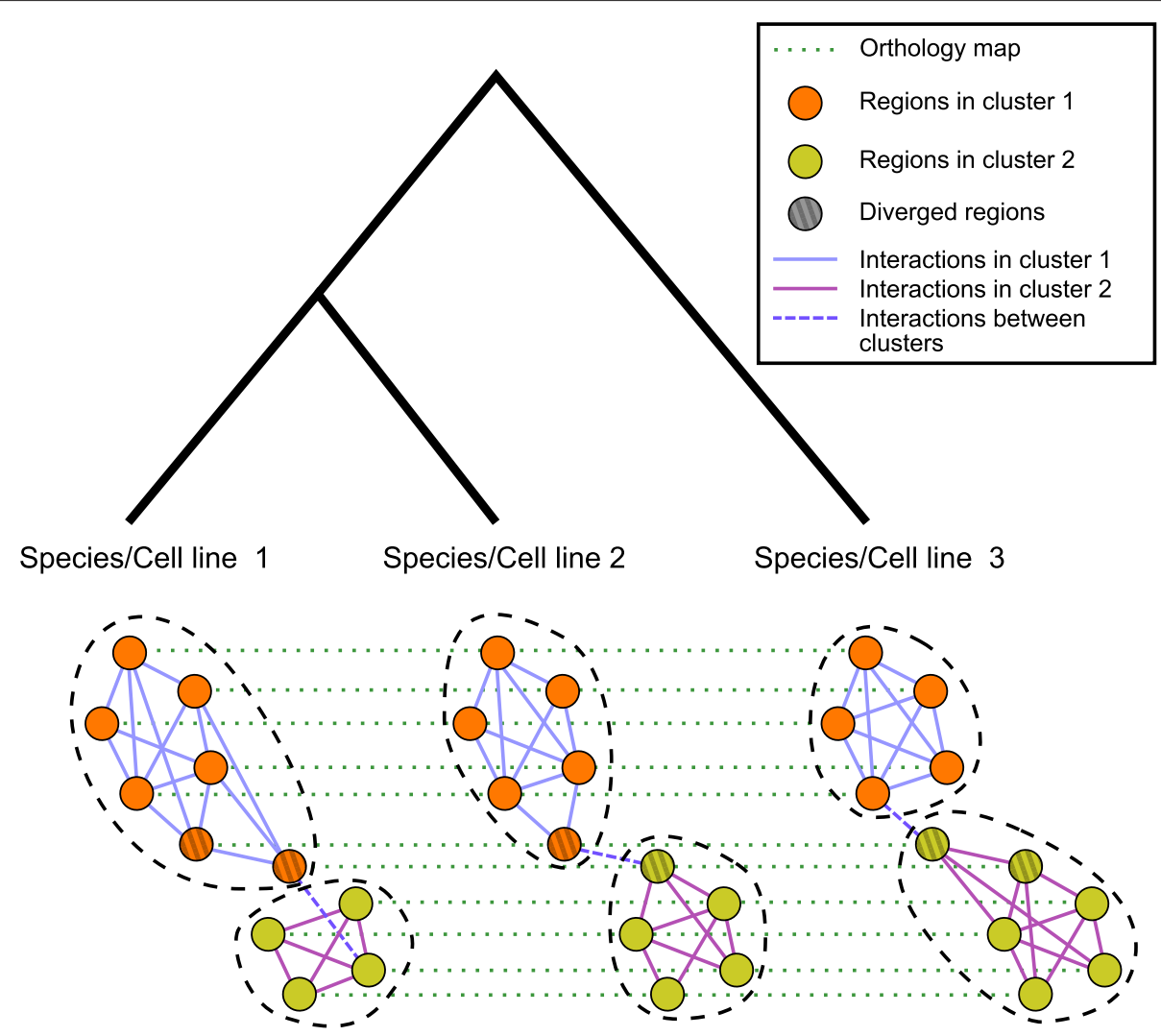

Fig. 3 Overview of Arboretum-Hi-C. Given $n$ graphs of genomic regions, each representing a Hi-C data set in $n$ species or cell lines, our method clusters the regions based on their interactions, while exploiting the relatedness (shown as the hierarchy) among the data sets. The figure shows an example of three $\mathrm{Hi}$-C data sets with two clusters. The example shows that some regions keep their cluster assignments in all species, while other regions (diverged regions) change their cluster based on changes in their interactions with other regions. Dashed lines represent a mapping of regions from one data set to another. For cell lines of the same species, this mapping is trivial as the same regions are studied. For multiple species, this mapping requires one to find orthologous regions between species

and mouse regions that constitute one-to-one orthologous regions (Methods). Results at a higher resolution (500 kbp) are described subsequently.

We considered two possible hierarchical relationships of these four data sets (Additional file 1: Figure S11 and Additional file 1: Methods) and used the probabilistic framework of Arboretum-Hi-C to select between these two trees. In one tree, the cell lines from the same species were closer to each other, and in the other, the embryonic cell lines from the two species were closer to each and the differentiated cell lines were closer to each other. We observed that the first tree, in which the $\mathrm{Hi}-\mathrm{C}$ data within a species were closer to each other, had a greater data likelihood (Additional file 1: Figure S11). Therefore, we performed our subsequent analysis with this tree topology.

Application of Arboretum-Hi-C to these four data sets identified ten clusters of interacting regions, several of which exhibited conserved patterns of interactions (Fig. 4). The multi-task clustering framework of Arboretum-Hi-C provides a correspondence between clusters of one cell line/species to the clusters of another cell line/species. That is, cluster $\mathrm{C} i$ from hESC would correspond to cluster $\mathrm{C} i$ of $\mathrm{mESC}$ (and all other data sets examined), where $i$ ranges from 0 to $k-1$. This correspondence or mapping of clusters between different data sets (as further described and validated below) enables a systematic comparison of patterns of interactions and the regions that participate in these interactions. We visually examined the patterns of these clusters based on the eigenvectors (Fig. 4a) as well as Spearman's correlation matrices for regions in each cluster (Fig. 4b). Several clusters exhibited conserved patterns of eigenvectors and interactions across all four data sets $(\mathrm{C} 1$ and $\mathrm{C} 2)$, while some clusters were more similar between cell lines of the same species [C6 (human) and C5], and some clusters captured similarity in the ESC state between species (C3 and C4).

To examine the extent of conservation at the region level, we examined these clusters in two ways. First, we extracted the core conserved set of regions by obtaining those regions that were in the same cluster in all species 


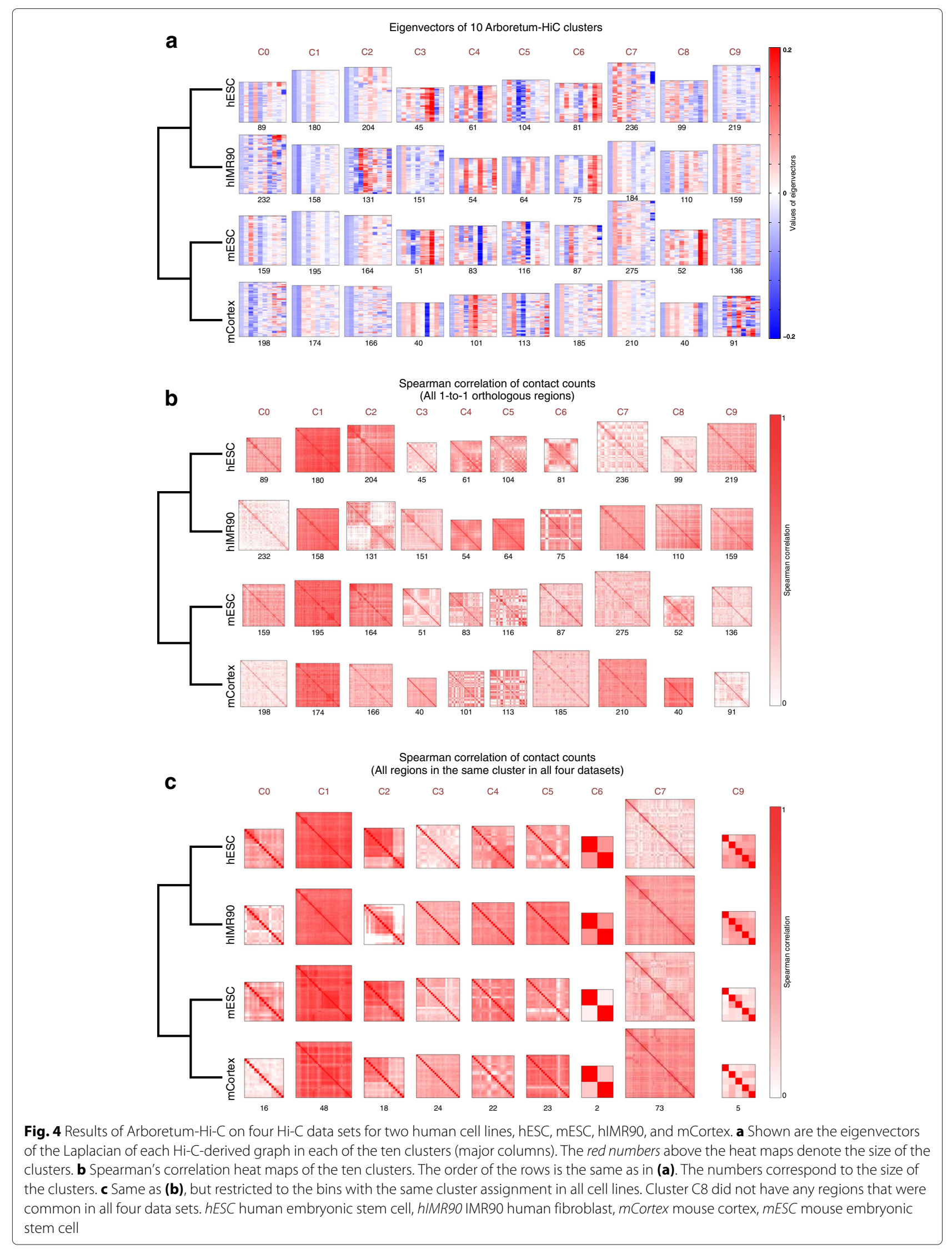


and cell lines (Fig. 4c). We observe a striking pattern of conservation of interactions in this conserved set of regions. For some clusters, this represented a high fraction of their elements ( $>30 \%$ for clusters C3, C4, and $\mathrm{C} 7)$, or a moderate fraction (10-30 \% for clusters $\mathrm{C} 0, \mathrm{C} 1$, $\mathrm{C} 2$, and $\mathrm{C5}$ ), while for some clusters this represented a small fraction ( $<10 \%$ for clusters C6, C8, and C9). Cluster C3 was the most conserved, with $44 \%$ of its regions in the conserved core set. Second, we compared the clusters, one pair of cell line/species at a time, using the significance of overlap of orthologous regions of one cluster from one species (or cell line), and another species (or cell line). We quantified the overlap in orthologous regions using the negative log of the hypergeometric test $P$ value as described in Roy et al. [30], and visualized them using red-blue heat maps (Fig. 5a), for every pair of species or cell lines. The off-diagonal elements of the heat map denote the shared chromosomal organization between clusters of different IDs, and the diagonal elements measure the extent of conservation between clusters of the same ID (Fig. 5a, red-blue heat maps). We found that between hESC and mESC (same cell type but different species), there were a larger number of strong red diagonal elements compared to hESC and mCortex.

To compare the extent of conservation between the clusters identified by Arboretum-Hi-C to clusters identified by applying spectral clustering to the data sets independently, we calculated the difference in the diagonal elements and off-diagonal elements for every pair of $\mathrm{Hi}-\mathrm{C}$ data sets over multiple random initializations of the algorithm. We find that using Arboretum-Hi-C there is greater conservation between clusters (Fig. 5b box plot of Arboretum-Hi-C clusters) of the matched cell lines ( $\mathrm{hESC}$ vs $\mathrm{mESC}$ ) than between different cell lines (hESC vs mCortex). In contrast, independent clustering of the $\mathrm{Hi}-\mathrm{C}$ data using non-multi-task spectral clustering did not discriminate between the cell lines and estimated a similar extent of conservation for both matched and different cell lines (Fig. 5b). Overall, the patterns of conservation and divergence from the non-multi-task clustering may not be as biologically meaningful as those from Arboretum-Hi-C.

To assess the extent to which conserved chromosomal modules exhibit similar regulatory signals and validate the mapping of clusters between data sets identified by Arboretum-Hi-C, we examined these clusters for enrichment of regulatory signals (Fig. 6). Arboretum-Hi-C $\mathrm{mESC}$ and hESC clusters of the same ID exhibited similar patterns of enrichment. In particular, clusters $\mathrm{C} 0, \mathrm{C} 1$, and $\mathrm{C} 2$, in both $\mathrm{hESC}$ and $\mathrm{mESC}$ were associated with generich, open chromatin, chromatin mark modified, LADdepleted regions (Fig. 6a, b). Similarly, clusters C3, C4, $\mathrm{C} 7, \mathrm{C} 8$, and $\mathrm{C} 9$ were gene poor and associated with LADs and repeat elements. SINEs tend to be associated with gene-rich, active chromatin, mark modified regions, while LINEs and LTRs are associated with LADs and gene-poor regions. Overall, we found that Arboretum-Hi-C clusters in both species could be grouped into clusters with high $(\mathrm{C} 0, \mathrm{C} 1$, and $\mathrm{C} 2)$ and low genomic activity $(\mathrm{C} 3, \mathrm{C} 4, \mathrm{C} 7, \mathrm{C} 8$, and $\mathrm{C9}$ ). While some clusters exhibited additional signal enrichment (e.g. mESC C9, H3K9me3, and DNase I), clusters with the same ID exhibited similar patterns of enrichment, despite not being completely orthologous, thus validating the correspondence of chromosomal cluster IDs of Arboretum-Hi-C.

To assess the effect of bin size in the definition of orthology mapping of the regions and the subsequent Arboretum-Hi-C analysis, we repeated our experiments at a higher resolution of $500 \mathrm{kbp}$, clustering a total of 2342 regions. As observed in the 1-Mbp case, we found conserved modules between human and mouse cell lines that could be matched based on their enrichment patterns (Additional file 1: Figure S12a, b and Additional file 2). Furthermore, we observed significant overlap between clusters obtained at 500-kbp resolution and 1-Mbp resolution (Additional file 1: Figure S12c), suggesting that changes in the bin size at this resolution (1 Mbp to $500 \mathrm{kbp}$ ) does not significantly affect the resulting clusters. To test whether intra-chromosomal interactions create a bias by overshadowing the interchromosomal interactions, we repeated our analysis after removing any interactions that are between regions of the same chromosome in human or mouse (Additional file 1: Methods). As in independent clustering, we observe significant overlap between clusters derived from interchromosomal interactions and clusters using both interand intra-chromosomal interactions (Additional file 1: Figure S13).

\section{Changes in chromosome contact modules between human and mouse cell lines}

We next examined module divergence between species and module dissimilarity between cell lines by inspecting the off-diagonal elements of the red-blue heat maps in Fig. 5a. This analysis relied on our characterization of clusters into high and low activity described in the previous section. We found that most of the module transitions were between clusters of the same type, that is, from low activity to low activity, or from high activity to high activity (Table 1). However, there were a few examples of transitions between modules with high and low genomic activity that we discuss below.

\section{Changes in high and low activity modules between species are associated with LADs and chromatin activity}

We found three examples of transitions between speciesspecific modules of different activities. One transition was between hESC C9 and mESC C0 involving 29 regions 

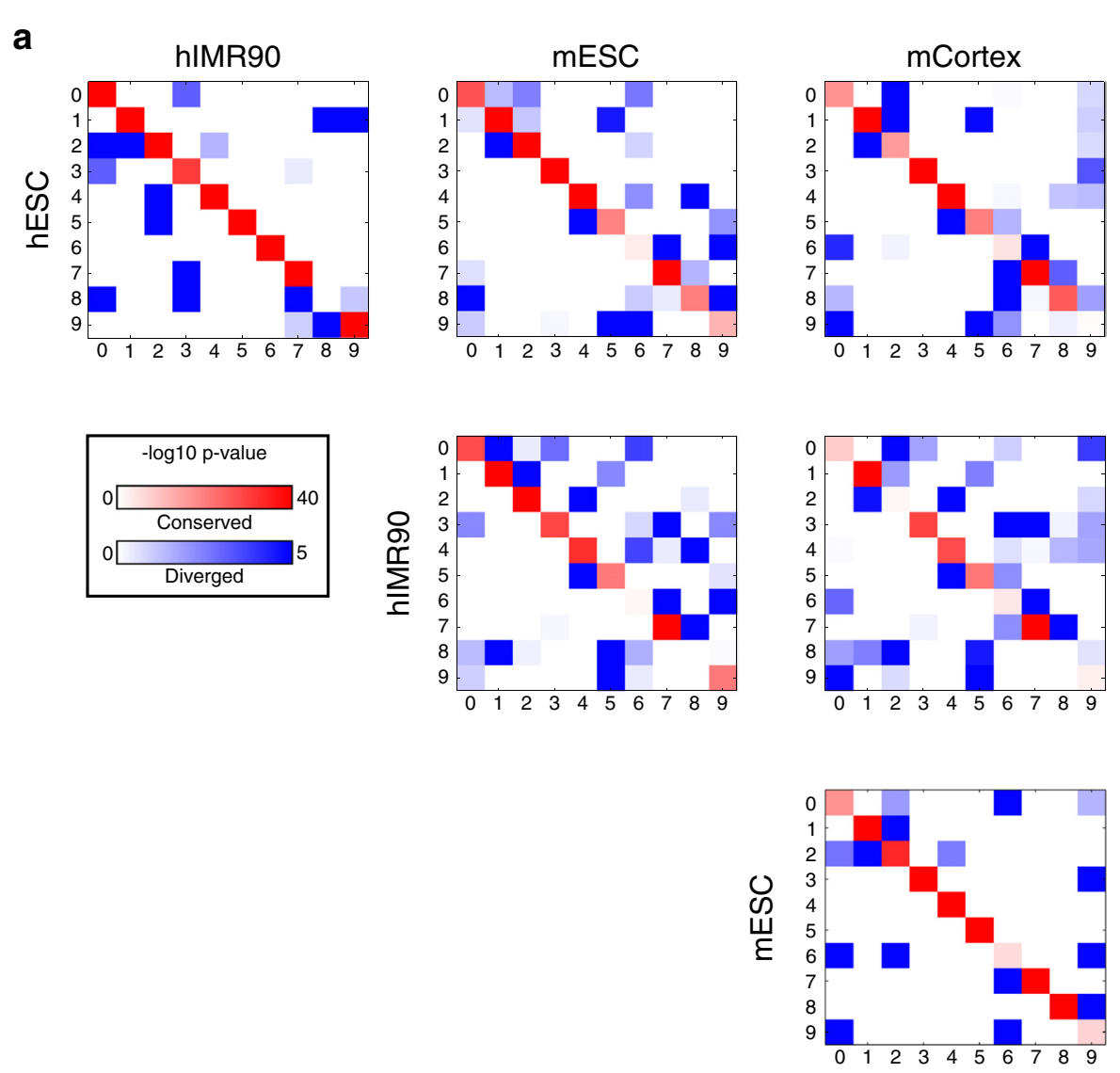

b

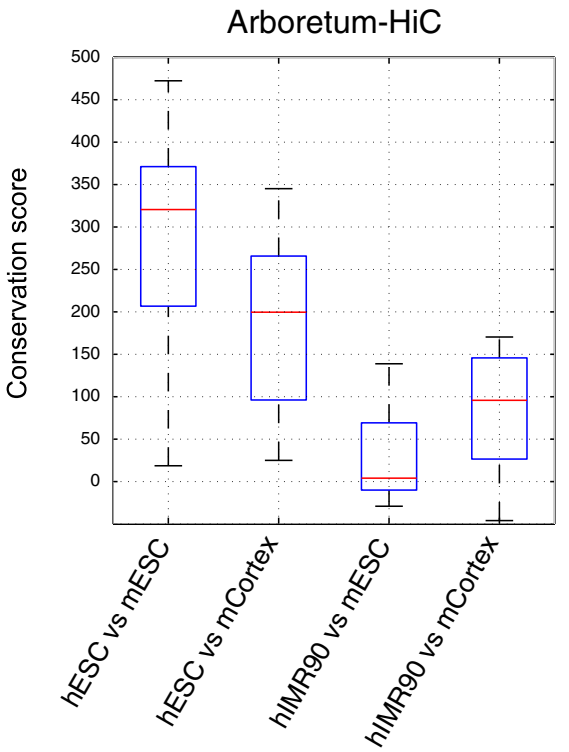

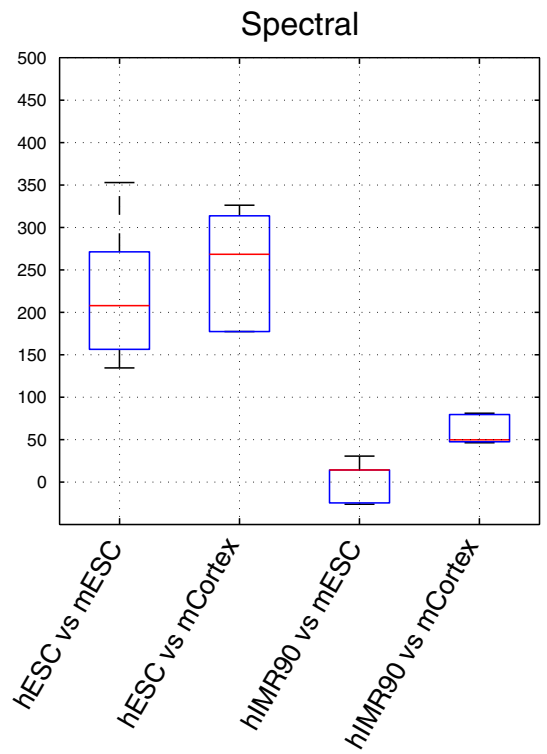

Fig. 5 a Overlap between clusters from four cell lines inferred by Arboretum-Hi-C. Each red-blue matrix shows the extent of similarity between clusters from pairs of cell lines or species as measured by $-\log _{10} P$ value of a hypergeometric test. Diagonal elements represent clusters of the same ID and are shown in red. Off-diagonal elements are shown in blue. The intensity of red and blue is proportional to the extent of similarity between pairs of clusters. $\mathbf{b}$ The distribution of conservation score between pairs of cell lines estimated from multiple random initializations of Arboretum-Hi-C and independent spectral clustering. The conservation score for Arboretum- $\mathrm{Hi}-\mathrm{C}$ was defined as the sum of diagonal elements minus off-diagonal elements of the matrices from (a). Because independent spectral clustering does not give a mapping of cluster assignments across data sets, we first matched cluster IDs based on maximal overlap of regions using the Hungarian algorithm (Methods). hESC human embryonic stem cell, hIMR90 IMR90 human fibroblast, mCortex mouse cortex, mESC mouse embryonic stem cell 


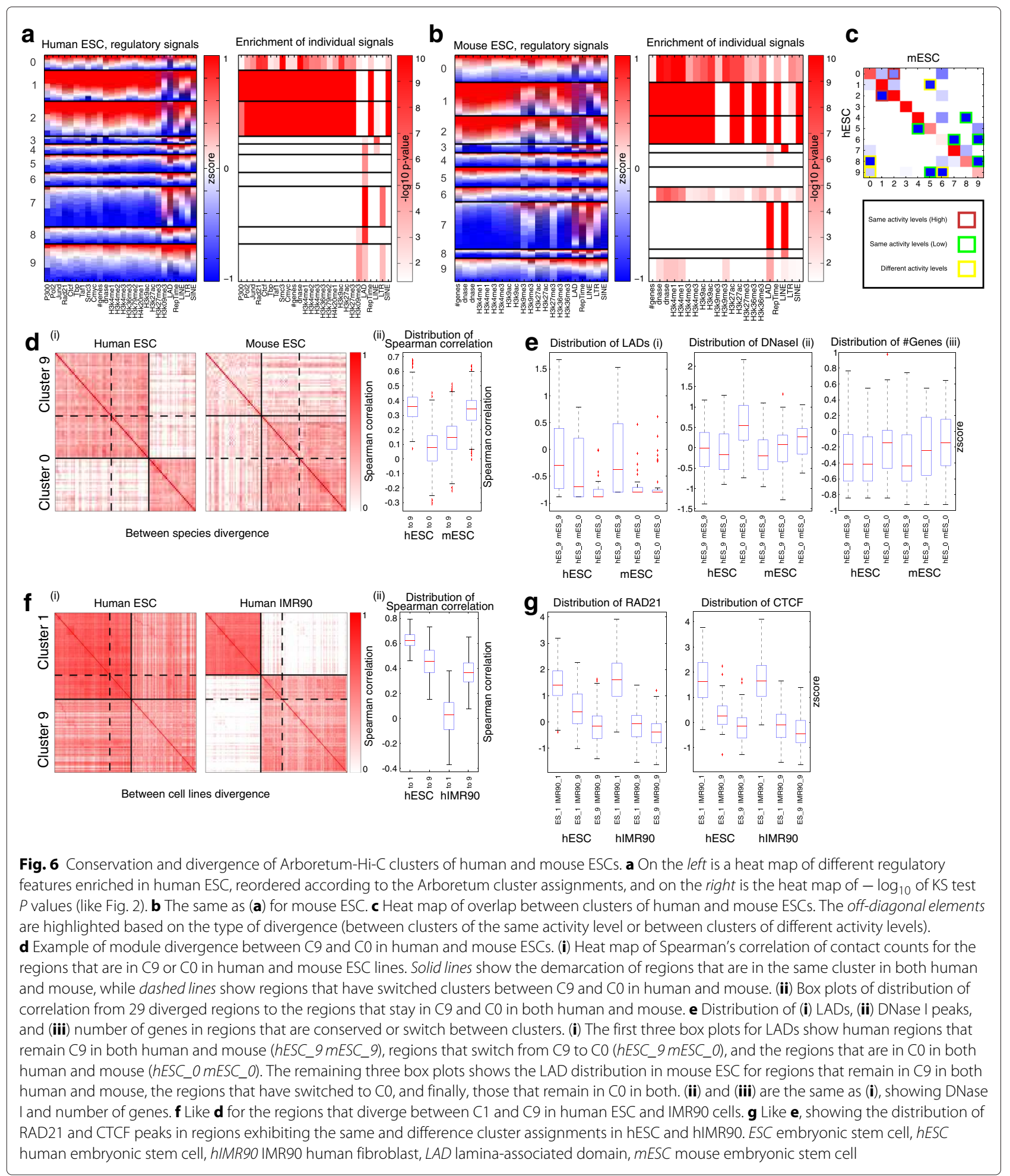

spanning a total $29 \mathrm{Mbp}$. The C9 cluster is associated with LADs, whereas $\mathrm{C} 0$ is associated with open chromatin and histone modification marks (Fig. 6a, b, c). Comparison of regulatory features of these 29 regions ( $h E S \_9 m E S \_0$ ) against regions that maintained their cluster assignment in C9 ( $\left.h E S \_9 m E S \_9\right)$ and C0 (hES_O mES_0) in hESC showed that these switched regions were less LAD-rich than the regions in cluster C9 (KS test $P<8.02 \times 10^{-2}$, Fig. 6e). In mESC, where these regions were assigned to $\mathrm{C} 0$, a cluster with high activity, they tended to have 
Table 1 Number of significant divergence events between pairs of clusters of different types in each pair of cell lines

\begin{tabular}{|c|c|c|c|}
\hline & & \multicolumn{2}{|c|}{ hIMR90 } \\
\hline \multirow{3}{*}{ hESC } & & High activity & Low activity \\
\hline & High activity & 3 & 1 \\
\hline & Low activity & 4 & 3 \\
\hline & & \multicolumn{2}{|c|}{ mESC } \\
\hline \multirow{3}{*}{ hESC } & & High activity & Low activity \\
\hline & High activity & 1 & 1 \\
\hline & Low activity & 2 & 7 \\
\hline \multirow{6}{*}{ hESC } & & \multicolumn{2}{|c|}{ mCortex } \\
\hline & & High activity & Low activity \\
\hline & High activity & 3 & 1 \\
\hline & Low activity & 2 & 5 \\
\hline & & \multicolumn{2}{|c|}{ mESC } \\
\hline & & High activity & Low activity \\
\hline \multirow{2}{*}{ hIMR90 } & High activity & 3 & 2 \\
\hline & Low activity & 0 & 7 \\
\hline \multirow{6}{*}{ hIMR90 } & & \multicolumn{2}{|c|}{ mCortex } \\
\hline & & High activity & Low activity \\
\hline & High activity & 3 & 2 \\
\hline & Low activity & 1 & 6 \\
\hline & & \multicolumn{2}{|c|}{ mCortex } \\
\hline & & High activity & Low activity \\
\hline \multirow{2}{*}{$\mathrm{mESC}$} & High activity & 2 & 1 \\
\hline & Low activity & 3 & 5 \\
\hline
\end{tabular}

hESC human embryonic stem cell, hIMR90 IMR90 human fibroblast, mCortex mouse cortex, mESC mouse embryonic stem cell

a lower propensity of LADs than other regions associated with C9 (KS test $P<8.66 \times 10^{-5}$ ), and more like other elements of $\mathrm{C} 0$. These regions exhibit a similar tendency for the number of genes and DNase I elements (Fig. 6e(ii), (iii)). A second transition, also between a high- and low-activity module, was from hESC C8 (low activity) to mESC CO (high activity, Fig. 6a, b, c) and included 21 regions. In both $\mathrm{hESC}$ and $\mathrm{mESC}$, these regions have significantly lower LAD content than the regions with conserved assignments to cluster $\mathrm{C} 8$ (KS test $P<4 \times 10^{-3}$, Additional file 1: Figure S14a). In addition, in hESCs, these regions have a significantly higher LAD content than regions that are in cluster $\mathrm{C} 0$ in both species (KS test $P<1 \times 10^{-4}$ ). Similarly, DNase I and gene count in human regions that switch are intermediate between the conserved members of $\mathrm{C} 8$ and $\mathrm{C} 0$ in both human and mouse (Additional file 1: Figure S14b, c). The third transition was in a different direction involving regions in a high-activity module in human (C1) and a module $\mathrm{C} 5$ in mouse, which was not significantly enriched for any signals in mouse, but is likely a lowactivity cluster based on the enrichment profile of the orthologous human C5. Although, the human regions that transitioned to module $\mathrm{C} 5$ in mouse did not exhibit a significantly different distribution in LADs, they exhibited a significantly depleted pattern of enrichment for DNase I (KS test $P<7.89 \times 10^{-3}$ ) and gene count (KS test $P<2.18 \times 10^{-2}$ when comparing diverged regions to $C 1$ in mouse, Additional file 1: Figure S14d,e). Overall, these results suggest that the regions that switch their chromatin interaction preference between species are associated with different one-dimensional signals than the regions that maintain their interaction preference between species.

\section{Changes in module assignment between cell lines are associated with CTCF and RAD2 1 binding sites}

In addition to transitions in modules between species, we found several examples of transitions between clusters with high and low activity among cell lines of the same species (five within human and four within mouse, Table 1). One example of such transitions is between cluster C9 (low activity) of hIMR90 and cluster C1 (high activity) of hESC. Figure $6 \mathrm{f}$ shows the pattern of correlation of contact counts for the regions in clusters $\mathrm{C} 1$ and $\mathrm{C} 9$ and regions that change their cluster assignment. To relate these transitions to the binding profiles of general transcription factors, we examined the distribution of binding of transcription factors measured in both cell lines, namely CEBPB, CTCF, MAFK, POLR2A, and RAD21 (Fig. 6g and Additional file 1: Figure S15). Among these transcription factors, CTCF and RAD21 appeared to discriminate $\mathrm{hESC}$ regions that remained in $\mathrm{C} 1$ and those that were in C9 in hIMR90 (KS test $P<6.04 \times 10^{-4}$ and $P<1.12 \times 10^{-4}$, respectively). Similarly, in hIMR90, these regions were more enriched than the regions that were in cluster C9 in both cell lines (KS test $P<6.20 \times$ $10^{-2}$ for CTCF and $P<3.05 \times 10^{-2}$ for RAD21). This differential enrichment suggests that CTCF and RAD21, which are known to be major players in chromosomal architecture and organization [31], likely contribute to cell-type-specific behavior between a differentiated and undifferentiated cellular state.

\section{Conclusions}

Chromosome conformation capture (3C) assays [2], such as $4 \mathrm{C}$ [32], 5C [33], and $\mathrm{Hi}-\mathrm{C}$ [8], as well as factorspecific ChIA-PET studies [34], are being increasingly applied to more cell types and species [3, 7, 14, 35-39]. 
Computational approaches for analyzing such data sets, and more importantly, comparing such maps across multiple tissues, are still in their infancy [20]. Here we performed a systematic analysis of graph-based and non-graph-based clustering methods for $\mathrm{Hi}-\mathrm{C}$ data. Our comparisons showed that graph-based clustering with different distance metrics tends to outperform nongraph-based clustering, suggesting that incorporating the graph-based nature of $\mathrm{Hi}-\mathrm{C}$ (and other $3 \mathrm{C}$ ) data is advantageous for clustering. We developed Arboretum-Hi-C, a novel graph-based multi-task clustering approach to find common and cell-line- and species-specific patterns of interacting chromosomal regions. The multi-task nature of our analysis framework enables us to uniformly map and compare clusters across multiple $\mathrm{Hi}-\mathrm{C}$ data sets. Furthermore, representing the relationship of these data sets as a tree enables us to study the extent of similarity of the corresponding species or cell lines. Simultaneous clustering of multiple data sets using the Arboretum-Hi-C framework showed that chromosome conformations in mESCs and hESCs are more similar to each other than between human and mouse differentiated cell states.

The ability to match clusters from one cell line or species to another becomes increasingly complicated as the number of cell lines or species increases. Arboretum-Hi-C addresses this challenge by using a multi-task clustering framework that also exploits the hierarchical relationships among cell types and species and where cluster IDs are tied to the topmost node in the hierarchy. Our approach provides a one-to-one mapping between clusters identified across multiple species or cell lines, which enables a systematic comparison of sets of regions across species and cell lines. We validated this one-to-one mapping in mouse and hESC lines by showing that the clusters of the same IDs are also enriched for similar regulatory signals (e.g. C1 of both hESCs and mESCs are associated with gene-poor LAD regions). We observed striking conservation between the modules inferred across the species for matched cell lines, which is consistent with a recent comparative study done in liver for four mammalian species [14]. We note that Arboretum-Hi-C is a data-driven approach and we used the data likelihood to decide between alternative tree topologies that could relate the $\mathrm{Hi}-\mathrm{C}$ data sets studied.

Our clustering approach also enabled us to study the context specificity of chromosomal interactions within and between species in a single unified framework. A change in cluster assignment between cell lines or between species suggests that those regions interact with other chromosomal regions. Such transitions are likely associated with the overall cell-line-specific or species-specific behaviors. We found that most of these changes are between modules with similar regulatory signals (that is, most transitions are between clusters with low activity and low activity, or high activity and high activity).

The occurrence of CTCF and RAD21 in regions that switch their chromosomal interaction cluster between cell lines is consistent with the role of these proteins as key determinants of the 3D organization of the genome. In particular, CTCF was shown to be associated with the divergence of TADs between species [14]. CTCF is also associated with cell-line-specific changes in TADs $[3,23]$. The presence of a LAD in regions that diverged their chromosomal contact preferences suggests a possible role of LADs in contributing raw material to the evolution of regulatory regions. However, with only two species, it is difficult to establish whether the changes in onedimensional signals are the cause or consequence of the topological reorganization. As the number of species with available $\mathrm{Hi}-\mathrm{C}$ data increases, we will be able to address these questions in a more principled manner. By comparing differentiated cells and undifferentiated cells from human and mouse, we were also able to examine the extent of conservation between matched cell types. We found that the modules identified in mESCs were more similar to hESCs. While this served as a useful validation of our data-driven clustering, having matched differentiated cell lines would greatly improve the comparative power of our approach.

We demonstrated our approach on relatively large regions $(1 \mathrm{Mbp})$ as well as variable-sized regions defined by TADs (Additional file 1: Figure S7) to enable the identification of large-scale chromosomal interactions that include both cis and trans interactions. However, our approach can also be applied in cis one chromosome at a time to identify TAD-like structures (Additional file 1: Methods and Additional file 1: Figure S10) as well as to find compartments (Additional file 1: Figure S9). These results suggest that this is a powerful and flexible clustering algorithm to identify known and novel chromosomal organizational units. Most of our analysis was done at a relatively coarse resolution, which remains fixed during the clustering procedure. An important extension is to have a flexible multi-resolution clustering algorithm that can adaptively select the distance measure depending upon the resolution.

In summary, we have performed a systematic analysis of different clustering methods for high-throughput $3 \mathrm{C}$ data sets that measure the $3 \mathrm{D}$ proximity of pairs of genomic regions. We also presented an algorithm to perform clustering across multiple species and identified patterns of significant conservation as well as species-specific and cell-line-specific divergence. As such $\mathrm{Hi}-\mathrm{C}$ maps become available for diverse cell types and species [9, 14, 40-42], methods such as ours will be increasingly useful for systematic comparisons to identify common and contextspecific properties of genome architecture, revealing 
principles governing the organization of chromatin and its impact on complex phenotypes.

\section{Methods \\ Data set description and pre-processing}

We used the publicly available $\mathrm{Hi}-\mathrm{C}$ data for two human cell lines (H1ES and IMR90) and two mouse cell lines (J1 ES and cortex) from Dixon et al. (GEO accession code GSE35156 [3]). Paired reads were aligned to the reference genomes (hg19 for human and $\mathrm{mm} 9$ for mouse), aggregated in different resolutions $(1 \mathrm{Mbp}, 500 \mathrm{kbp}$, and $100 \mathrm{kbp}$ bins), and then normalized to correct for known biases using iterative correction and eigenvector decomposition (ICE) [21]. The data sets are deeply sequenced with 600-900 million reads, enabling us to examine both intra- and inter-chromosomal interactions. After binning and normalization, we had a total 2755,5465 , and 27,179 bins in human at $1-\mathrm{Mbp}, 500-\mathrm{kbp}$, and 100-kbp resolution, respectively. In mouse, we had 2469,4901 , and 24,213 bins at $1-\mathrm{Mbp}, 500-\mathrm{kbp}$, and 100-kbp resolution, respectively.

\section{Clustering algorithms for one data set}

We considered three classes of clustering algorithms: hierarchical, $k$-means, and spectral clustering, each with five different distance metrics: Euclidean distance, Pearson's correlation, Spearman's correlation, contact counts, and $\log _{2}$ contact counts. We further adapted the distance to suit each method as described below.

\section{Determining the number of clusters}

We treat the number of clusters as an input parameter for the clustering algorithms examined. For our analysis, we inspected the interaction patterns obtained from spectral clustering. Specifically, we permuted the adjacency matrix to create a randomized graph, and compared the distribution of eigenvalues of the Laplacian of the original graph and the randomized graph. We observed that the difference in eigenvalues between the random and the original graph was not significant beyond the first 15 , and therefore, we set 15 to be the upper limit on the number of clusters. We learned $k \in 2,5,10,15$ clusters and manually inspected their contact count profiles and decided that $k=10$ provides the best results.

\section{Hierarchical clustering}

To perform hierarchical clustering with contact count and $\log _{2}$ of contact counts as distances, we subtracted the maximum value of the count (or $\log _{2}$ count) matrix from the given matrix. For the other three distance metrics, given the $\log _{2}$ of the genome-wide normalized contact count matrix, we calculated the distance of all pairs of bins using the pdist function in Matlab. To define the clusters, we used average linkage (using the linkage function) and the cluster function (with option maxclust set to $k$ ) to find $k$ clusters. Using Euclidean distance and
Spearman's correlation, we observed a number of very small clusters $(<10$ elements) and one very large cluster. Because assessing statistical enrichment of signals is difficult for such small clusters, we applied a post-processing step to obtain more balanced clusters. Specifically, we kept partitioning the largest cluster until we reached $k$ clusters with at least ten elements, and then added the clusters with less than ten elements to the largest cluster.

\section{k-means}

For Euclidean distance and Pearson's correlation, we used the kmeans function in Matlab. This function does not provide clustering using Spearman's correlation distance, so we implemented $k$-means with 1 -Spearman's correlation as the distance measure. To cluster with contact counts and $\log _{2}$ of contact counts, we also implemented a modified version of $k$-means similar to the $k$-means algorithm described in Yaffe et al. [19].

\section{Spectral clustering}

Our spectral clustering algorithm is motivated by the fact that the Hi-C interaction map can be viewed as a weighted graph with vertices representing regions. The weight of the edge between a pair of regions can correspond to the contact count between the two regions (or $\log _{2}$ of contact count) or a more indirect but global measure of similarity of the interaction profile of those regions (e.g. using a Spearman's or Pearson's correlation). A graph-based framework was recently shown to capture several topological properties of chromosome organization in yeast [22] and improve chromatin-mark-based genome annotation [23], suggesting that a graphical representation serves as a powerful representation for $\mathrm{Hi}-\mathrm{C}$ data. Spectral clustering is a graph-clustering method that uses the eigenvectors of the Laplacian of a graph for clustering [24, 25, 43].

We used the algorithm described by Rohe et al. [24], which is based on clustering the eigenvectors corresponding to the largest eigenvalues of the graph Laplacian matrix. For each variant of similarity measure, we created a different weighted graph, with the weight representing the similarity measure. Let $A$ denote an $n \times n$ adjacency matrix, where $n$ is the total number of regions. Let $A(i, j)$ denote the edge weight between regions $i$ and $j$. For the Euclidean distance, $A(i, j)=M-e_{i, j}$ where $e_{i, j}$ is the Euclidean distance between row $i$ and row $j$ of the $\log _{2}$ of the normalized contact count matrix, and $M$ is the maximum observed Euclidean distance. Thus, two regions that have a large value of $e_{i, j}$ will be less similar to each other than two regions with a small value of $e_{i, j}$. For the Pearson's or Spearman's correlation, $A(i, j)=c_{i, j}$ if $c_{i, j} \geq 0$, and $A(i, j)=0$ otherwise, where $c_{i, j}$ is the correlation between row $i$ and row $j$ of the $\log _{2}$ of the normalized contact count matrix. For the normalized contact counts and $\log _{2}$ of normalized contact counts, $A(i, j)$ was set to the 
corresponding count or $\log _{2}$ of the contact count between regions $i$ and $j$. The Laplacian of the graph is defined as $L=D^{-1 / 2} A D^{-1 / 2}$ where $D$ is a diagonal matrix with each element $D(i, i)=\sum_{k} a_{i, k}$. Thus, the Laplacian gives a normalized degree distribution of all vertices. We used the eigs function in Matlab to calculate the eigenvectors and eigenvalues of the Laplacian. Once we have the eigenvectors, the $k$-means algorithm is used to cluster the matrix $\mathbf{X}=\left\{X_{1}, X_{2}, \ldots, X_{k}\right\}$ where $X_{i}$ is a column vector in $R^{n}$ and $X_{1}, X_{2}, \ldots, X_{k}$ are the first $k$ eigenvectors of $L$, corresponding to the $k$ largest eigenvalues of $L$.

\section{Description of cluster evaluation criteria}

We used five different statistical measures to assess the quality of our clusters.

\section{Davies-Bouldin index}

We defined the DBI as

$$
\mathrm{DBI}=\frac{1}{k} \sum_{i=1}^{k} \max _{j \neq i} D_{i, j}
$$

where

$$
D_{i, j}=\frac{\bar{d}_{i}+\bar{d}_{j}}{d_{i, j}} .
$$

In the traditional definition of DBI, $\bar{d}_{i}$ is defined as the distance of elements in cluster $i$ to its center, and $d_{i, j}$ is the distance of the centers of clusters $i$ and $j$. Because in some of our clustering methods we do not have a center for the clusters, we defined $\bar{d}_{i}$ as the average distance of all pairs of elements in cluster $i$, and $d_{i, j}$ as the average distance of pairs of elements where one element was in cluster $i$ and the second element was in cluster $j$. We used 1-Spearman's correlation as the distance metric.

\section{Silhouette index}

We defined the SI as

$$
\mathrm{SI}=\frac{1}{k} \sum_{i=1}^{k} \frac{1}{\left|C_{i}\right|} \sum_{j \in C_{i}} s_{j}
$$

where

$$
s_{j}=\frac{b_{j}-a_{j}}{\max \left\{a_{j}, b_{j}\right\}}
$$

and $a_{j}$ is defined as the average distance of element $j$ to all other members of its own cluster $\left(C_{i}\right)$, and $b_{j}$ is the average distance of element $j$ to the members of the second best cluster (the cluster other than $C_{i}$ with lowest average distance to element $j$ ). We used 1-Spearman's correlation as the distance metric.

\section{Delta contact counts}

This measure was defined on the log of the contact count matrix. For each cluster $C_{i}$, let $\operatorname{in}_{i}$ denote the average $\log$ of contact counts for pairs of regions in that cluster, and out $_{i}$ denote the average log of contact counts for pairs of regions where one region is in cluster $C_{i}$ and the other region is not. We define the delta contact count, $D$, as

$$
D=\frac{1}{k} \sum_{i=1}^{k} \operatorname{in}_{i}-\text { out }_{i} .
$$

We expect that for a good cluster, the pairs of regions within the cluster should have higher contact counts. Therefore, the higher the value of $D$, the higher the quality of the clusters.

\section{Number of enriched clusters}

For each cluster and each genomic signal, we used the KS test to compare the distribution of the values of the given signal for the regions inside and outside the cluster. We test whether the values inside the cluster are significantly higher than values outside the cluster. If the $P$ value returned by the KS test was lower than 0.05 , we considered that cluster enriched for the given signal. We counted the number of clusters that were enriched for at least one signal. To calculate the $P$ value of the KS test we used the kstest2 function of Matlab with the smaller switch.

\section{ANOVA test}

To test how well our clusters can separate the regulatory signals, we performed a one-way ANOVA test for each given signal and the cluster assignments for all regions examined. We used the anoval function of Matlab, and used the sum of $-\log$ of $P$ values over all the given signals to rank the clustering methods.

\section{Arboretum-Hi-C: a multi-task clustering approach for multiple $\mathrm{Hi}-\mathrm{C}$ data sets}

To perform multi-task clustering between the four cell lines, we first found a one-to-one mapping between orthologous 1-Mbp (and 500-kbp) bins between human and mouse and extracted contact count matrices corresponding to orthologous regions (see below). Next, we calculated the eigenvectors of the Laplacian as described above (spectral clustering with Spearman's correlation). We ran Arboretum on the eigenvectors that had an orthologous region in the other species as described in detail below.

\section{Orthology mapping between regions in human and mouse}

To define the orthologous pairs of regions between human and mouse at a particular resolution $r$, we split the genome of each species into contiguous regions of $r$ base pairs (1 Mbp or $500 \mathrm{kbp}$ ). We used a stringent criterion to define the orthology by requiring these regions to satisfy two filters. First, we used blastn with the option -evalue $1 \mathrm{E}-5$ to align these regions to each other. For each pair of regions $h_{i}$ and $m_{j}$ from human and mouse, we sum the number of base pairs aligned between the two regions 
$\left[A\left(h_{i}, m_{j}\right)\right]$. For a region $h_{i}$ in human, we find the region from mouse $m_{j}$ with the longest alignment to it: $m_{j}=$ $\operatorname{argmax}_{m_{j^{\prime}}} A\left(h_{i}, m_{j^{\prime}}\right)$. Similarly, for a region $m_{j}$ in mouse, we find the region $h_{i}$ in human with the longest alignment to it. We accept a pair of regions $\left(h_{i}, m_{j}\right)$ as orthologous if they are reciprocal hits.

Our second filter used whole-genome alignments specified in chain files from the UCSC Genome Bioinformatics website (http://genome.ucsc.edu/) [44, 45]. We read the chain files and for each chain of alignments, we iterate over the alignment segments and add the length of the aligned segment to the corresponding pair of regions in human and mouse. For each region in human, we select the region in mouse with the largest sum of aligned segments (and vice versa for mouse to human) and selected the best reciprocal hits. We further filter these orthologous pairs by removing any pair with a sum of segments $<0.1 r$ ( $1 \mathrm{Mbp}$ or $500 \mathrm{kbp}$ ). There is a significant agreement between the orthology mapping produced by the two approaches ( $\sim 5 \%$ of the pairs produced from blastn are also in the other map). Our final set of orthologous mappings for input to Arboretum-Hi-C was obtained by taking the intersection of orthologous pairs from the above two filtering approaches. This results in 1318 orthologous regions between human and mouse at $1-\mathrm{Mbp}$ resolution and 2342 regions at 500-kbp resolution.

\section{Arboretum algorithm for multi-task clustering}

Arboretum was developed to cluster multiple expression data sets, one from each species, while exploiting the gene and species tree phylogenies in the clustering using a probabilistic framework [30]. This approach favors orthologous genes having the same cluster assignment between species subject to the support in the data. Instead of clustering the expression of the genes, here we use Arboretum to cluster the eigenvectors of the Laplacian of the graphs produced from the $\mathrm{Hi}-\mathrm{C}$ data; and rather than clustering the eigenvectors of each cell line separately, we cluster multiple $\mathrm{Hi}-\mathrm{C}$ data sets simultaneously. To run Arboretum, we need a mapping between the elements that are being clustered (e.g. 1-Mbp regions) and also a tree structure to capture the relationships between data sets from different species and cell lines. We experimented with different tree structures and selected the one that gave us better likelihood (Additional file 1: Methods and Additional file 1: Figure S11).

\section{Comparison of cluster similarity between pairs of cell lines/species}

We used the hypergeometric test to compute the significance of similarity between pairs of clusters for each pair of cell lines/species. Given the matrix of $-\log _{10}$ of the hypergeometric test's $P$ value for pairs of clusters, the conservation score was defined as the sum of the diagonal elements (clusters with matched IDs) minus the sum of the off-diagonal elements (clusters with different IDs). Because independent spectral clustering does not provide a mapping of cluster assignments across data sets, we first used the Hungarian algorithm [46] to find the best one-toone matching between the two given cluster assignments that maximizes the overlap between matched clusters, and using this matching we calculated the conservation score (as described above).

\section{Compartment identification and comparison to spectral clustering clusters}

To define compartments, we followed the procedure described in Lieberman et al. [8]. We used the raw contact counts (before applying ICE for normalization) and calculated the genome-wide average contact count $I_{s}$ for all possible genomic distances $s$. For each chromosome, we defined a matrix $M$ by dividing the contact counts of pairs of regions at distance $s$ by $I_{s}$. We computed the Spearman's correlation for entries in $M$ and took the first principal component of this correlation matrix. We defined the two compartments based on positive and negative values of the first principal component.

To compare the spectral clusters to the two compartments in each chromosome, we used two different measures: the $F$ score and the Rand index. To calculate the $F$ score, we first count the number of pairs of regions that were in the same cluster in spectral clusters $s$, the number of pairs of regions that were in the same compartment $c$, and the number of pairs of regions that were grouped together in both methods $o$. We defined precision $p=o / s$, recall $r=o / c$, and $F$ score

$$
f=\frac{2 p r}{p+r}
$$

We defined the Rand index as

$$
R=\frac{o+b}{\left(\begin{array}{l}
n \\
2
\end{array}\right)}
$$

where $b$ is the number of pairs of regions that were in different modules in both methods and $n$ is the number of regions.

\section{One-dimensional genomic signals for interpretation of clusters}

To interpret the clusters obtained by the different clustering methods examined, we obtained a number of genomic signals representing binding profiles of transcription factors, chromatin state, and density of genes and repeat elements. We aggregated these signals into fixed-size bins (1 Mbp or $500 \mathrm{kbp}$ ) or into variable-sized bins defined by TADs. Below we refer to both fixed- and variable-sized bins. 


\section{Number of genes}

We downloaded the annotation files for hg19 and $\mathrm{mm} 9$ assembly from the Ensembl website [47]. We aggregated the genes in a bin and counted the number of genes in each and used these counts as a signal for each bin.

\section{Transcription factors}

We used the transcription factor narrow peak files from ENCODE [42] for CEBPB, CMYC, CTCF, JUND, MAFK, P300, POL2, POLR2A, RAD21, SMC3, TAF1, and TBP for hESCs, and CEBPB, CTCF, MAFK, POLR2A, and RAD21 for the IMR90 cell line. We aggregated the peaks in each bin and counted the number of peaks in each bin. The number of peaks per bin was used as a signal for the bin.

\section{DNase I and histone marks}

We used peak files from ENCODE [42] for DNase I, H3k4me1, H3k4me2, H3k4me3, H3k9ac, H3k9me3, H3k27ac, H3k27me3, H3K36me3, H3k79me2, and H4k20me1 in hESC, and DNase I, H3k4me1, H3k4me3, H3k9ac, H3k9me3, H3k27ac, H3k27me3, and H3k36me3 in $\mathrm{mESC}$. We aggregated the peaks in a bin and counted the number of peaks in each bin and used these counts as features.

\section{LADs and replication timing}

We downloaded LADs from Meuleman et al. [28] and used the percentage of $1-\mathrm{Mbp}$ bins covered with LADs as a feature. We also downloaded replication timing data from Ryba et al. [48] for hESC, and from Hiratani et al. [49] for mESCs, and used the average of the replication timing ratio ( $\log _{2}$ of early to late) in each bin as a feature value. The $\mathrm{LAD}$ and replication timing data were aligned to hg18, and we used liftover to map them to hg19 coordinates [44].

\section{Other sequence features}

We downloaded SINE, LINE, and LTR repeats from the UCSC Genome Bioinformatics website (http://genome. ucsc.edu/) [45]. For each type of repeat, we counted the number of repeats in each bin and used these counts as features.

\section{Availability of data and materials}

The scripts, programs, and data sets used in this study are available at http://zenodo.org/record/49767 and https:// bitbucket.org/roygroup/arboretum-hic (under GPLv3). The data sets supporting the conclusions of this article are included within the article (and its additional files).

\section{Ethics approval}

Not applicable.

\section{Additional files}

Additional file 1: Supplemental data including supplementary text, Figs. S1-S15 and Table S1. (PDF $8740 \mathrm{~kb}$ )

Additional file 2: The resulting cluster assignments reported in this study, and the feature matrices used for enrichment analysis. (XLS $4270 \mathrm{~kb}$ )

\section{Competing interests}

The authors declare that they have no competing interests.

\section{Authors' contributions}

SR designed this study. FA processed and provided the data and helped with the interpretation of the results. AF performed the experiments. SR and AF wrote the manuscript. All authors read and approved the final manuscript.

\section{Acknowledgments}

We thank Rupa Sridharan for helpful discussions.

\section{Funding}

FA was supported by a Computing Research Association CIFellows award [National Science Foundation (NSF) award Computing Innovation Fellowship (CIF) 1136996] and by the Institute Leadership Professorship Fund from La Jolla Institute for Allergy and Immunology. This research was also funded in part by National Institute of Allergy and Infectious Diseases (NIAID), National Institute of Environmental Health Sciences (NIEHS), NINDS (National Institute of Neurological Disorders and Stroke), National Institute on Deafness and Other Communication Disorders (NIDCD), and NIAAA (National Institute on Alcohol Abuse and Alcoholism) of the National Institutes of Health $(\mathrm{NIH})$ under award number U54AI117924. The content is solely the responsibility of the authors and does not necessarily represent the official views of $\mathrm{NIH}$. This work is also funded in part by an NSF Career award (NSF Division of Biological Infrastructure(DBI): 1350677) and a Sloan Foundation research fellowship to SR.

\section{Author details}

${ }^{1}$ Department of Computer Sciences, University of Wisconsin, Madison, WI 53717, USA. ${ }^{2}$ La Jolla Institute for Allergy and Immunology, 9420 Athena Circle, La Jolla, CA 92037 USA. ${ }^{3}$ Wisconsin Institute for Discovery, University of Wisconsin, Madison, WI 53717, USA. ${ }^{4}$ Department of Biostatistics and Medical Informatics, University of Wisconsin, Madison, WI 53717, USA.

Received: 27 February 2016 Accepted: 22 April 2016

Published online: 27 May 2016

References

1. de Wit E, de Laat W. A decade of 3C technologies: insights into nuclear organization. Genes Dev. 2012;26(1):11-24. doi:10.1101/gad.179804.111.

2. Dekker J, Rippe K, Dekker M, Kleckner N. Capturing chromosome conformation. Science. 2002;295(5558):1306-11. doi:10.1126/science.1067799.

3. Dixon JR, Selvaraj S, Yue F, Kim A, Li Y, Shen Y, et al. Topological domains in mammalian genomes identified by analysis of chromatin interactions. Nature. 2012;485(7398):376-80. doi:10.1038/nature11082.

4. Duan Z, Andronescu M, Schutz K, Mcllwain S, Kim YJ, Lee C, et al. A three-dimensional model of the yeast genome. Nature. 2010;465(7296): 363-7. doi:10.1038/nature08973.

5. Jin F, Li Y, Dixon JR, Selvaraj S, Ye Z, Lee AY, et al. A high-resolution map of the three-dimensional chromatin interactome in human cells. Nature. 2013;503(7475):290-4. doi:10.1038/nature12644

6. Kalhor R, Tjong H, Jayathilaka N, Alber F, Chen L. Genome architectures revealed by tethered chromosome conformation capture and population-based modeling. Nat Biotech. 2012;30(1):90-8. doi:10.1038/nbt.2057.

7. Li G, Ruan X, Auerbach RK, Sandhu KS, Zheng M, Wang P, et al. Extensive promoter-centered chromatin interactions provide a topological basis for transcription regulation. Cell. 2012;148(12):84-98. doi:10.1016/j.cell.2011.12.014.

8. Lieberman-Aiden E, van Berkum NL, Williams L, Imakaev M, Ragoczy T, Telling $A$, et al. Comprehensive mapping of long-range interactions 
reveals folding principles of the human genome. Science. 2009;326(5950): 289-93. doi:10.1126/science.1181369.

9. Rao SSP, Huntley MH, Durand NC, Stamenova EK, Bochkov ID, Robinson JT, et al. A 3D map of the human genome at kilobase resolution reveals principles of chromatin looping. Cell. 2014;159(7):1665-80. doi:10.1016/j.cell.2014.11.021.

10. Sexton T, Yaffe E, Kenigsberg E, Bantignies F, Leblanc B, Hoichman M, et al. Three-dimensional folding and functional organization principles of the Drosophila genome. Cell. 2012;148(3):458-72. doi:10.1016/j.cell.2012.01.010.

11. Dekker J, Marti-Renom MA, Mirny LA. Exploring the three-dimensional organization of genomes: interpreting chromatin interaction data. Nat Rev Genet. 2013;14(6):390-403. doi:10.1038/nrg3454.

12. Tanizawa H, Iwasaki O, Tanaka A, Capizzi JR, Wickramasinghe P, Lee M, et al. Mapping of long-range associations throughout the fission yeast genome reveals global genome organization linked to transcriptional regulation. Nucleic Acids Res. 2010;38(22):8164-77. doi:10.1093/nar/gkq955.

13. Ma W, Ay F, Lee C, Gulsoy G, Deng X, Cook S, et al. Fine-scale chromatin interaction maps reveal the cis-regulatory landscape of human lincRNA genes. Nat Meth. 2015;12(1):71-8. doi:10.1038/nmeth.3205.

14. Vietri Rudan M, Barrington C, Henderson S, Ernst C, Odom DT, Tanay A, et al. Comparative Hi-C reveals that CTCF underlies evolution of chromosomal domain architecture. Cell Rep. 2015;10(8):1297-1309.

15. Chambers EV, Bickmore WA, Semple CA. Divergence of mammalian higher order chromatin structure is associated with developmental loci. PLoS Comput Biol. 2013;9(4):1003017. doi:10.1371/journal.pcbi.1003017.

16. Dixon JR, Jung I, Selvaraj S, Shen Y, Antosiewicz-Bourget JE, Lee AY, et al. Chromatin architecture reorganization during stem cell differentiation. Nature. 2015;518(7539):331-6. doi:10.1038/nature14222.

17. Ay F, Bailey TL, Noble WS. Statistical confidence estimation for Hi-C data reveals regulatory chromatin contacts. Genome Res. 2014;24(6): 999-1011. doi:10.1101/gr.160374.113.

18. Witten DM, Noble WS. On the assessment of statistical significance of three-dimensional colocalization of sets of genomic elements. Nucleic Acids Res. 2012;40(9):3849-55. doi:10.1093/nar/gks012.

19. Yaffe E, Tanay A. Probabilistic modeling of $\mathrm{Hi}-\mathrm{C}$ contact maps eliminates systematic biases to characterize global chromosomal architecture. Nat Gen. 2011;43(11):1059-65. doi:10.1038/ng.947.

20. Ay F, Noble WS. Analysis methods for studying the $3 \mathrm{D}$ architecture of the genome. Genome Biol. 2015;16(1):1-15. doi:10.1186/s13059-015-0745-7.

21. Imakaev M, Fudenberg G, McCord RPP, Naumova N, Goloborodko A, Lajoie BR, et al. Iterative correction of Hi-C data reveals hallmarks of chromosome organization. Nat Methods. 2012;9(10):999-1003. doi:10.1038/nmeth.2148.

22. Wang H, Duggal G, Patro R, Girvan M, Hannenhalli S, Kingsford C. Topological properties of chromosome conformation graphs reflect spatial proximities within chromatin. In: Proceedings of the International Conference on Bioinformatics, Computational Biology and Biomedical Informatics BCB'13. New York: ACM; 2013. p. 306. doi:10.1145/2506583.2506633

23. Libbrecht MW, Ay F, Hoffman MM, Gilbert DM, Bilmes JA, Noble WS. Joint annotation of chromatin state and chromatin conformation reveals relationships among domain types and identifies domains of cell-type-specific expression. Genome Res. 2015;25(4):544-57. doi:10.1101/gr.184341.114.

24. Rohe K, Qin T, Yu B. Co-clustering for directed graphs: the Stochastic co-Blockmodel and spectral algorithm Di-Sim. arXiv preprint arXiv:1204.2296. 2015.

25. von Luxburg U. A tutorial on spectral clustering. Stat Comput. 2007;17(4): 395-416. doi:10.1007/s11222-007-9033-Z.

26. Jiang D, Tang C, Zhang A. Cluster analysis for gene expression data: a survey. Knowl Data Eng IEEE Trans. 2004;16(11):1370-86. doi:10.1109/tkde.2004.68.

27. Kundaje A, Meuleman W, Ernst J, Bilenky M, Yen A, et al. Integrative analysis of 111 reference human epigenomes. Nature. 2015;518(7539): 317-30. doi:10.1038/nature14248.

28. Meuleman W, Peric-Hupkes D, Kind J, Beaudry JB, Pagie L, Kellis M, et al. Constitutive nuclear lamina-genome interactions are highly conserved and associated with A/T-rich sequence. Genome Res. 2013;23(2):270-80 doi:10.1101/gr.141028.112.
29. Caruana R. Multitask learning: Kluwer Academic Publishers vol. 28; 1997, pp. 41-75. doi:10.1023/a\%253a1007379606734.

30. Roy S, Wapinski I, Pfiffner J, French C, Socha A, Konieczka J, et al. Arboretum: reconstruction and analysis of the evolutionary history of condition-specific transcriptional modules. Genome Res. 2013;23(6): 1039-50. doi:10.1101/gr.146233.112.

31. Merkenschlager M, Odom DT. CTCF and cohesin: linking gene regulatory elements with their targets. Cell. 2013;152(6):1285-97. doi:10.1016/j.cell.2013.02.029.

32. Simonis M, Klous $P$, Splinter E, Moshkin $Y$, Willemsen $R$, de Wit E, et al. Nuclear organization of active and inactive chromatin domains uncovered by chromosome conformation capture-on-chip (4C). Nat Genet. 2006;38(11):1348-54. doi:10.1038/ng1896.

33. Dostie J, Richmond TA, Arnaout RA, Selzer RR, Lee WL, Honan TA, et al. Chromosome conformation capture carbon copy (5C): a massively parallel solution for mapping interactions between genomic elements. Genome Res. 2006;16(10):1299-309. doi:10.1101/gr.5571506.

34. Fullwood MJ, Liu MH, Pan YF, Liu J, Xu H, Mohamed YB, et al. An oestrogen-receptor- $\alpha$-bound human chromatin interactome. Nature. 2009;462(7269):58-64. doi:10.1038/nature08497.

35. Bantignies F, Roure V, Comet I, Leblanc B, Schuettengruber B, Bonnet J, et al. Polycomb-dependent regulatory contacts between distant Hox loci in Drosophila. Cell. 2011;144(2):214-26. doi:10.1016/j.cell.2010.12.026.

36. Heidari N, Phanstiel DH, He C, Grubert F, Jahanbani F, Kasowski M, et al. Genome-wide map of regulatory interactions in the human genome. Genome Res. 2014;24(12):1905-17. doi:10.1101/gr.176586.114.

37. Sanyal A, Lajoie BR, Jain G, Dekker J. The long-range interaction landscape of gene promoters. Nature. 2012;489(7414):109-13. doi:10.1038/nature11279.

38. Schoenfelder S, Sexton T, Chakalova L, Cope NF, Horton A, Andrews S, et al. Preferential associations between co-regulated genes reveal a transcriptional interactome in erythroid cells. Nat Genet. 2009;42(1): 53-61. doi:10.1038/ng.496.

39. Wang KC, Yang YW, Liu B, Sanyal A, Corces-Zimmerman R, Chen Y, et al. A long noncoding RNA maintains active chromatin to coordinate homeotic gene expression. Nature. 2011;472(7341):120-4. doi:10.1038/nature09819.

40. Ho JWK, Jung YL, Liu T, Alver BH, Lee S, Ikegami K, et al. Comparative analysis of metazoan chromatin organization. Nature. 2014;512(7515): 449-52. doi:10.1038/nature13415.

41. modENCODE Consortium, Roy S, Ernst J, Kharchenko PV, Kheradpour P, Negre $N$, et al. Identification of functional elements and regulatory circuits by Drosophila modENCODE,. Science. 2010;330(6012):1787-97. doi:10.1126/science.1198374.

42. The ENCODE Project Consortium. An integrated encyclopedia of DNA elements in the human genome. Nature. 2012;489(7414):57-74. doi:10.1038/nature11247.

43. Dhillon IS, Guan Y, Kulis B. Kernel k-means: spectral clustering and normalized cuts. In: Proceedings of the 10th ACM SIGKDD International Conference on Knowledge Discovery and Data Mining. KDD '04. New York: ACM; 2004. p. 551-6. doi:10.1145/1014052.1014118.

44. Hinrichs AS, Karolchik D, Baertsch R, Barber GP, Bejerano G, Clawson H, et al. The UCSC genome browser database: update 2006. Nucleic Acids Res. 2006;34(Database issue):590-8. doi:10.1093/nar/gkj144.

45. Rosenbloom KR, Armstrong J, Barber GP, Casper J, Clawson H, Diekhans M, et al. The UCSC genome browser database: 2015 update. Nucleic Acids Res. 2014. doi:10.1093/nar/gku1177.

46. Kuhn HW. The Hungarian method for the assignment problem. Naval Res Logistics. 1955;2(1-2):83-97. doi:10.1002/nav.3800020109.

47. Cunningham F, Amode MR, Barrell D, Beal $K$, Billis $K$, Brent $S$, et al. Ensembl 2015. Nucleic Acids Res. 2015;43(Database issue):662-9. doi:10.1093/nar/gku1010.

48. Ryba T, Hiratani I, Lu J, Itoh M, Kulik M, Zhang J, et al. Evolutionarily conserved replication timing profiles predict long-range chromatin interactions and distinguish closely related cell types. Genome Res. 2010;20(6):761-70. doi:10.1101/gr.099655.109.

49. Hiratani I, Ryba T, Itoh M, Rathjen J, Kulik M, Papp B, et al. Genome-wide dynamics of replication timing revealed by in vitro models of mouse embryogenesis. Genome Res. 2010;20(2):155-69. doi:10.1101/gr.099796.109. 